\title{
Mean-Field Solution of the Mixed Spin-2 and Spin-5/2 Ising Ferrimagnetic System with Different Single-Ion Anisotropies
}

\author{
Fathi Abubrig \\ Department of Physics, Faculty of Science, Elmergeb University, Zliten, Libya \\ Email: dr_fathiomar@yahoo.com
}

Received December 26, 2012; revised February 13, 2013; accepted February 20, 2013

Copyright (C) 2013 Fathi Abubrig. This is an open access article distributed under the Creative Commons Attribution License, which permits unrestricted use, distribution, and reproduction in any medium, provided the original work is properly cited.

\begin{abstract}
The mixed spin-2 and spin-5/2 Ising ferrimagnetic system with different anisotropies $\left(D_{A} / z|J|\right)$ for the spin-2 and $\left(D_{B} / z|J|\right)$ for the spin-5/2 is studied by the use of the mean-field theory based on the Bogoliubov inequality for the free energy. First, the ground state phase diagram of the system at zero temperature is obtained on the $\left(D_{A} / z|J|, D_{B} / z|J|\right)$ plane. Topologically, different kinds of phase diagrams are achieved by changing the temperature and the values of the single ion anisotropies $D_{A} / z|J|$ and $D_{B} / z|J|$. Besides second-order transition lines, first order phase transition lines terminating at tricritical points, are found. The existence and dependence of a compensation temperature on single-ion anisotropies is also investigated.
\end{abstract}

Keywords: Mixed Spin; Ising Model; Ferrimagnetic; Sublattice Magnetization; Tricritical Points

\section{Introduction}

In the last two decades, much attention has been paid to the study of the magnetic properties of two-sublattice mixed-spin ferrimagnetic Ising systems, because they are well adapted to consider some types of ferrimagnetism, namely the molecular-based magnetic materials [1-3] which have less translational symmetry than their singlespin counterparts since they consist of two interpenetrating sublattices and have increasing interest. In a ferrimagnetic material, the different temperature dependences of the sublattice magnetizations raise the possibility of the existence of a compensation temperature: a temperature below the critical point where the total magnetization is zero [4]. This interesting behaviour has important applications in the field of thermomagnetic recording [5, 6]. For this reason, in recent years, there have been many theoretical studies on the magnetic properties of systems formed by two sublattices with different spins and with different crystal field interactions.

One of the earliest and simplest of these models to be studied was the mixed-spin Ising system consisting of spin-1/2 and spin-S $(\mathrm{S}>1 / 2)$ in a uniaxial crystal field.
The model for different values of $\mathrm{S}(\mathrm{S}>1 / 2)$ has been investigated by acting on honey-comb lattice [7-9], as well as on Bethe lattice [10,11]), mean field approximation [12], effective field theory with correlations [13-17], cluster variational theory [11], renormalization-group technique [18] and Monte-Carlo simulation [19-21].

It should be mentioned that the effects of different sublattice crystal-field interactions on the magnetic properties of the mixed spin-1 and spin-3/2 Ising ferromagnetic system with different single-ion anisotropies have been investigated with the use of an effective field theory $[22,23]$, mean field theory [24], a cluster variational method [25] and Monte Carlo simulation [26]. Recently, The attention was devoted to the high order mixed spin ferrimagnetic systems (mixed spin-3/2 and spin-2 ferrimagnetic system mixed spin- 2 and spin- $5 / 2$ ferrimagnetic system and mixed spin-3/2 and spin-5/2 system) in order to construct their phase diagrams in the temperatureanisotropy plane and to consider their magnetic properties. Bobak and Dely investigated the effect of single-ion anisotropy on the phase diagram of the mixed spin-3/2 and spin-2 Ising system by the use of a mean-field theory based on the Bogoliubov inequality for the free energy 
[27]. Albayrak also studied the mixed spin-3/2 and spin-2 Ising system with two different crystal-field interactions on Bethe lattice by using the exact recursion equations [28]. Bayram Deviren et al. have used the effective field theory to study the magnetic properties of the ferrimagnetic mixed spin-3/2 and spin-2 Ising model with crystal field in a longitudinal magnetic field on a honeycomb and a square lattice [29]. We should mention that an early attempt to study the mixed-spin- 2 and spin- $5 / 2$ system on a honeycomb lattice was made by Kaneyoshi and co-workers [30] within the frame work of the EFT. Nakamura [31,32] applied Monte Carlo (MC) simulations to study the magnetic properties of a mixed spin-2 and spin-5/2 system on a honeycomb lattice. Li et al. [33, 34] studied the magnetic properties of the mixed spin-2 and spin-5/2 system on a layered honeycomb lattice by a multisublattice green-function technique to investigate the magnetic properties of a mixed

$\mathrm{AFe}^{\mathrm{II}} \mathrm{Fe}^{\mathrm{III}}\left(\mathrm{C}_{2} \mathrm{O}_{4}\right)_{3}\left[\mathrm{~A}=\mathrm{N}\left(n-\mathrm{C}_{n} \mathrm{H}_{2 n+1}\right), n=3,5\right]$ and to consider the compensation behaviour of the system. Wei and co-worker [35] examined the internal energy, specific heat and initial susceptibility of the mixed spin-2 and spin-5/2 ferrimagnetic system with an interlayer coupling by the use of the EFT with correlations. Albayrak [36] studied the critical behaviour of the mixed spin2 and spin-5/2 Ising ferrimagnetic system on Bethe lattice. And he also examined the critical and the compensation temperatures of the mixed spin-2 and spin-5/2 Ising ferrimagnetic system on Bethe lattice by using the exact recursion equations. Keskin and Ertas [37] investigated the Existence of a dynamic compensation temperature of a mixed spin-2 and spin-5/2 Ising ferrimagnetic system in an oscillating field.

In this paper, we studied the effects of two different single-ion anisotropies in the phase diagram and in the compensation temperature of the mixed spin-2 and spin$5 / 2$ Ising ferrimagnetic system within the theoretical framework of the mean-field theory and we found some outstanding features in the temperature dependences of total and sublattice magnetizations.

The outline of this work is as follows. In Section 2, we define the model and present the mean-field theory based on the Bogoliubov inequality for the Gibbs free energy and then, we describe a Landau expansion of the free energy in the order parameter. In Section 3, we present the results and the discussion about the phase diagrams and compensation temperature for various values of the single-ion anisotropies, as well as the temperature dependences of the magnetizations in some particular cases. Finally, in Section 4, we present our conclusions.

\section{The Model and Calculation}

We consider a mixed Ising spin-2 and spin-5/2 system consisting of two sublattices $A$ and $B$, which are arranged alternately. The sublattice $A$ are occupied by spins $S_{i}$, which take the spin values of $\pm 2, \pm 1,0$, while the sublattice $B$ are occupied by spins $S_{j}$, which take the spin values of $\pm 5 / 2, \pm 3 / 2,1 / 2$. In each site of the lattice, there is a single-ion anisotropy $\left(D_{A}\right.$ in the sublattices $A$ and $D_{B}$ in the sublattice $B$ ) acting in the spin-2 and spin-5/2. The Hamiltunian of the system according to the mean-field theory is given by

$$
H=-J \sum_{(i, j)} S_{i}^{A} S_{j}^{B}-D_{A} \sum\left(S_{i}^{A}\right)^{2}-D_{B} \sum\left(S_{j}^{B}\right)^{2},
$$

where the first summation is carried out only over nearest-neighbor pairs of spins on different sublattices and $J$ is the nearest-neighbour exchange interaction.

The most direct way of deriving the mean-field theory is to use the variation principle for the Gibbs free energy,

$$
G(H) \leq \Phi \equiv G_{0}\left(H_{0}\right)+\left\langle H-H_{0}\right\rangle_{0},
$$

where $G(H)$ is the true free energy described by Hamiltonian given in the relation $(1), G_{0}(H)$ is the free energy described by the trial Hamiltonian $H_{0}$ which depends on variational parameters and $\langle\cdots\rangle_{0}$ denotes a thermal average over the ensemble defined by $H_{0}$.

Depending on the choice of the trial Hamiltonian, one can construct approximate methods of different accuracy. However, owing to the complexity of the problem, we consider in this work the simple choice of $H_{0}$, namely:

$$
\begin{aligned}
H_{0}= & -\sum_{i \in A}\left[\gamma_{A} S_{i}^{A}+D_{A}\left(S_{i}^{A}\right)^{2}\right] \\
& -\sum_{j \in B}\left[\gamma_{B} S_{j}^{B}+D_{B}\left(S_{j}^{B}\right)^{2}\right],
\end{aligned}
$$

where $\gamma_{A}$ and $\gamma_{B}$ are the two variational parameters related to the molecular fields acting on the two different sublattices, respectively. Through this approach, we found the free energy and the equations of state (sublattice magnetization per site $m_{A}$

$$
\begin{aligned}
g= & \frac{\Phi}{N}=\frac{-1}{2 \beta} \ln \left[1+2 \exp \left(4 \beta D_{A}\right) \cosh \left(2 \beta \gamma_{A}\right)+2 \exp \left(\beta D_{A}\right) \cosh \left(\beta \gamma_{A}\right)\right] \\
& -\frac{1}{2 \beta} \ln \left[2 \exp \left(\frac{25}{4} \beta D_{B}\right) \cosh \left(\frac{5}{2} \beta \gamma_{B}\right)+2 \exp \left(\frac{9}{4} \beta D_{B}\right) \cosh \left(\frac{3}{2} \beta \gamma_{B}\right)+2 \exp \left(\frac{1}{4} \beta D_{B}\right) \cosh \left(\frac{1}{2} \beta \gamma_{B}\right)\right] \\
& -\frac{1}{2} z J m_{A} m_{B}+\frac{1}{2} \gamma_{A} m_{A}+\frac{1}{2} \gamma_{B} m_{B},
\end{aligned}
$$


where $g=\frac{\Phi}{N}, \beta=\frac{1}{k_{B} T}, N$ is the total number of sites of the lattice and $z$ is the coordination number.

The sublattice magnetization per site $m_{A}$ and $m_{B}$ are defined by $m_{A}=\left\langle S_{i}^{A}\right\rangle_{0}$ and $m_{B}=\left\langle S_{j}^{B}\right\rangle_{0}$, thus

$$
m_{A}=\frac{2 \sinh \left(2 \beta \gamma_{A}\right)+\exp \left(-3 \beta D_{A}\right) \sinh \left(\beta \gamma_{A}\right)}{\cosh \left(2 \beta \gamma_{A}\right)+\exp \left(-3 \beta D_{A}\right) \cosh \left(\beta \gamma_{A}\right)+0.5 \exp \left(-4 \beta D_{A}\right)}
$$

and

$$
m_{B}=\frac{1}{2}\left[\frac{5 \sinh \left(\frac{5}{2} \beta \gamma_{B}\right)+3 \exp \left(-4 \beta D_{B}\right) \sinh \left(\frac{3}{2} \beta \gamma_{B}\right)+\exp \left(-6 \beta \gamma_{B}\right) \sinh \left(\frac{1}{2} \beta \gamma_{B}\right)}{\cosh \left(\frac{5}{2} \beta \gamma_{B}\right)+\exp \left(-4 \beta D_{B}\right) \cosh \left(\frac{3}{2} \beta \gamma_{B}\right)+\exp \left(-6 \beta D_{B}\right) \cosh \left(\frac{1}{2} \beta \gamma_{B}\right)}\right] .
$$

Now, by minimizing the free energy (4) with respect to $\gamma_{A}$ and $\gamma_{B}$, we obtain

The mean-field properties of the present model are then given by Equations (4)-(7). Since the Equations (5)(7) have in general several solutions for the pair $\left(m_{A}, m_{B}\right)$, the stable phase will be the one which minimizes the free energy. When the system undergoes the second-order transition from an ordered state $\left(m_{A} \neq 0, m_{B} \neq 0\right)$, to the paramagnetic state $\left(m_{A}=0, m_{B}=0\right)$, this part of the phase diagram can be determined analytically.

Because the magnetizations $m_{A}$ and $m_{B}$ are very small in the neighborhood of second-order transition

$$
\gamma_{A}=z J m_{B}, \gamma_{B}=z J m_{A} .
$$

point, we can expand Equations (4)-(6) to obtain a Landau-like expansion.

$$
g=g_{0}+a m_{A}^{2}+b m_{A}^{4}+c m_{A}^{6}+O\left(m_{A}^{8}\right),
$$

where the expansion coefficients are given by

$$
\begin{aligned}
& g_{0}=-\frac{1}{2 \beta} \ln \left[\left(1+X_{A}+Y_{A}\right)\left(X_{B}+Y_{B}+Z_{B}\right)\right], \\
& a=\frac{1}{2 \beta}\left[\frac{t^{2}}{4} a_{1}-\frac{t^{2}}{8} a_{2}-\frac{t^{4}}{32} a_{1}^{2} b_{1}\right], \\
& b=\frac{1}{2 \beta}\left[\frac{t^{4}}{768} a_{1}^{2} c_{1}+\frac{t^{3}}{192} c_{2} a_{1} a_{2}+\frac{t^{2}}{96} c_{3}\right],
\end{aligned}
$$

with

$$
\begin{gathered}
a_{1}=\frac{9 R_{1}+R_{2}+25}{R_{2}+R_{1}+1}, \quad a_{2}=\frac{4 X_{A}+Y_{A}}{X_{A}+Y_{A}+1}, \\
a_{3}=\frac{81 R_{1}+R_{2}+625}{R_{1}+R_{2}+1}, \quad a_{4}=\frac{16 X_{A}+Y_{A}}{X_{A}+Y_{A}+1}, \\
a_{5}=\frac{729 R_{1}+R_{2}+15625}{R_{1}+R_{2}+1}, \quad a_{6}=\frac{64 X_{A}+Y_{A}}{X_{A}+Y_{A}+1}, \\
b_{1}=\frac{25 X_{B}+9 Y_{B}+Z_{B}}{X_{B}+Y_{B}+Z_{B}}, \quad b_{2}=\frac{625 X_{B}+81 Y_{B}+Z_{B}}{X_{B}+Y_{B}+Z_{B}}, \\
b_{3}=\frac{15625 X_{B}+729 Y_{B}+Z_{B}}{X_{B}+Y_{B}+Z_{B}}, \\
c_{2}=\frac{t^{3}}{2}\left(3 a_{1}^{2}-a_{3}\right), \quad c_{3}=\frac{t^{2}}{4}\left(3 b_{1}^{2}+4 a_{3}-12 a_{1}^{2}-b_{2}\right), \\
c_{4}=\frac{t^{2}}{4}\left(15 b_{1} b_{2}-b_{1}-b_{2}-30\right), \\
c_{5}=\frac{t^{3}}{4}\left(a_{5}+30 a_{1}^{3}-15 a_{3} a_{1}\right),
\end{gathered}
$$

where

$$
c_{6}=\frac{t^{4}}{12}\left(15 a_{1}^{6} a_{2} a_{4}-a_{1}^{6} a_{6}-30\left(a_{1}^{2} a_{2}\right)^{3}\right),
$$

$$
\begin{gathered}
X_{A}=2 \exp \left(4 \beta D_{A}\right), \quad X_{B}=2 \exp \left(25 \beta D_{B} / 4\right), \\
Y_{A}=2 \exp \left(\beta D_{A}\right), \quad Y_{B}=2 \exp \left(9 \beta D_{B} / 4\right), \\
Z_{B}=2 \exp \left(\beta D_{B} / 4\right), \quad R_{1}=2 \exp \left(-4 \beta D_{A}\right), \\
R_{2}=2 \exp \left(-6 \beta D_{A}\right) .
\end{gathered}
$$

In this way, critical and tricritical points are determined according to the following routine;

1) Second-order transition lines when $a=0$ and $b>0$;

2) Tricritical points when $a=b=0$, and $c>0$;

3) The first-order transition lines are determined by comparing the corresponding Gibbs free energies of the various solutions of Equations (5) and (6) for the pair $\left(m_{A}, m_{B}\right)$. Even so, we have also checked that $c>0$ in all $T, D_{A}, D_{B}$ space. The critical behaviour is the same for both ferromagnetic $(J>0)$ and ferrimagnetic $(J<0)$ systems, because the coefficients $a, b$ and $c$ are even 
functions of $J$. On the other hand, the total magnetization per site.

$$
M=\frac{1}{2}\left(m_{A}+m_{B}\right)
$$

and the signs of sublattice magnetizations $m_{A}$ and $m_{B}$ are different, therefore, a compensation temperature

$T_{k}\left(T_{k}<T_{c}\right)$ at which the total magnetization is equal to zero may be exist in the system, although $m_{A} \neq 0$ and $m_{B} \neq 0$. In our paper we shall prove whether the present mixed-spin system can exhibit a compensation point or not.

\section{Results and Discussions}

\subsection{Phase Diagrams}

The ground-state phase diagram is easily determined from Hamiltonian (1) by comparing the ground-state energies of the different phases and is shown in Figure 1. At zero temperature, we find six phases with different values of $\left\{m_{A}, m_{B}, q_{A}, q_{B}\right\}$, namely the ordered ferrimagnetic phases

$$
\begin{aligned}
& O_{1}=\left\{-2, \frac{5}{2}, 4, \frac{25}{4}\right\}, O_{2}=\left\{-2, \frac{3}{2}, 4, \frac{9}{4}\right\}, \\
& O_{3}=\left\{-2, \frac{1}{2}, 4, \frac{1}{4}\right\}, \quad O_{4}=\left\{-1, \frac{5}{2}, 1, \frac{25}{4}\right\}, \\
& O_{5}=\left\{-1, \frac{3}{2}, 1, \frac{9}{4}\right\}, \quad O_{6}=\left\{-1, \frac{1}{2}, 1, \frac{1}{4}\right\},
\end{aligned}
$$

and three disordered phases

$$
D_{1}=\left\{0,0,0, \frac{25}{4}\right\}, D_{2}=\left\{0,0,0, \frac{9}{4}\right\}, D_{3}=\left\{0,0,0, \frac{1}{4}\right\},
$$

where the parameters $q_{A}$ and $q_{B}$ are defined by:

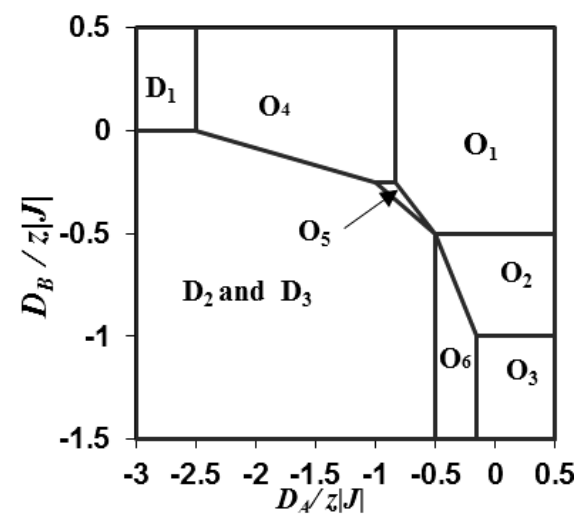

Figure 1. Ground-state phase diagram of mixed spin-2 and spin-5/2 Ising ferrimagnetic system with the coordination number $z$ and different single-ion anisotropies $D_{A}$ and $D_{B}$. The nine phases: ordered $\mathrm{O}_{1}, \mathrm{O}_{2}, \mathrm{O}_{3}, \mathrm{O}_{4}, \mathrm{O}_{5}, \mathrm{O}_{6}$ and disordered $D_{1}, D_{2}, D_{3}$ are separated by lines of first-order transitions.

$$
q_{A}=\left\langle S_{i}^{A}\right\rangle^{2}, q_{B}=\left\langle S_{j}^{B}\right\rangle^{2}
$$

\subsection{Temperature Phase Diagrams}

In Figures $\mathbf{2}$ and 3, the phase diagrams of the mixed spin-2 and spin-5/2 Ising ferrimagnetic system are shown in the $\left(D_{A} / z|J|, k_{B} T_{c} / z|J|\right)$ and $\left(D_{B} / z|J|, k_{B} T_{c} / z|J|\right)$ planes for some selected values of $D_{B} / z|J|$ for spin-5/2 and $D_{A} / z|J|$ for spin-2, respectively. The solid and light dotted lines are used for the second and first-order transition, respectively, the heavy dashed curve represents the positions of tricritical points. The second-order phase transition lines are easily obtained from Equations (10) and (11) by setting $a=0$ and $b>0$.

The tricritical points (the critical points at which the phase transitions change from second to first order) are determined from Equations (10) and (11) by setting $a=b$ $=0$, however, the first-order phase transitions must be determined by comparing the corresponding Gibbs free energies of the various solutions of (5) and (6) for the pair $\left(m_{A}, m_{B}\right)$.

In Figure 2, we note that the value of the critical temperature increases when $D_{B} / z|J|$ and $D_{A} / z|J|$ increases. Above each second-order lines the system is in the paramagnetic state, while below them is in the ferrimagnetic state. We note that the system gives only second-order phase transitions (solid lines) for all the values of $D_{A} / z|J|>-0.4661$ and the phase diagram is topologically equivalent to that of the spin- $5 / 2$ Blume-Capel model which does not include any tricritical point.

For the values of $-2.3315 \leq D_{A} / z|J| \leq-0.4661$ the system includes second-order phase transition lines (solid lines) at higher temperatures, first-order phase transition lines (light dotted lines) at lower temperatures and a curve of tricritical (heavy dashed lines) points separates

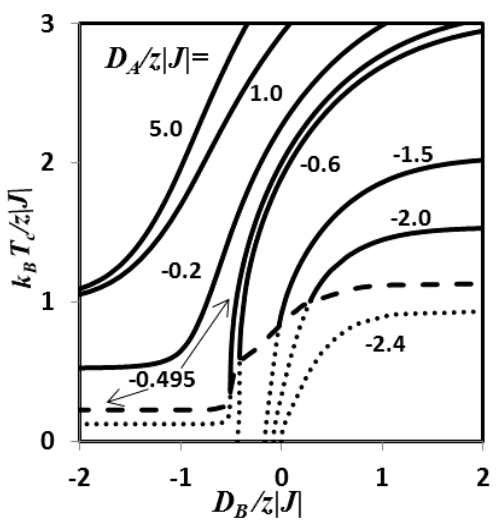

Figure 2. Phase diagram in the $\left(D_{B}, T\right)$ plane for the mixedspin Ising ferrimagnet with the coordination number $z$, when the value of $D_{B} / z|J|$ is changed. The solid and dotted lines, respectively, indicate second and first-order phase transitions, while the heavy dashed line represents the positions of tricritical points. 
the second and the first-order critical lines.

When $-2.5<D_{A} / z|J|<-2.3315$, the system gives only first-order phase transition lines.

In Figure 3, the phase diagrams of $\left(k_{B} T_{c} / z|J|\right)$ versus $D_{A} / z|J|$ are shown for selected values of $D_{B} / z|J|$ From this figure, it is clear that in regions of high temperatures, for all positive or negative values of, and for any value of $D_{B} / z|J|$, the phase diagram shows only second-order phase transitions.

When $D_{B} / z|J| \geq 1.4650$, all the second-order lines end in the same tricritical point given by

$\left(D_{A} / z|J|, k_{B} T_{3 c} / z|J|\right)=(-2.3315,1.1360)$ and when

$D_{B} / z|J| \leq-0.8450$, all the second-order lines end in the same tricritical point given by $\left(D_{A} / z|J|, k_{B} T_{3 c} / z|J|\right)=$ $(-0.4661,0.2272)$. From this figure, we also note that for $D_{B} / z|J| \rightarrow+\infty$, the mixed spin Ising system behaves like a two-levels system since the spin-5/2 behaves like $S_{j}^{B}= \pm 5 / 2$ and the coordinates $\left(D_{A} / z|J|, k_{B} T_{3 c} / z|J|\right)$ of the tricritical point are $(-2.3315,1.1360)$.

On the other hand, for $D_{B} / z|J| \rightarrow-\infty$, the $S_{j}^{B}= \pm 5 / 2$ and $S_{j}^{B}= \pm 3 / 2$ states are suppressed and the system becomes equivalent to mixed spin-1/2 and spin-2 Ising model with tricritical point located at $\left(D_{A} / z|J|, k_{B} T_{3 c} / z|J|\right)=(-0.4661,0.2272)$. For this reason, the coordinates of the tricritical point in the limit of large positive $D_{B} / z|J|$ are five times higher than those for large negative $D_{B} / z|J|$.

\subsection{Magnetization Curves}

Thermal behaviour of the sublattice magnetizations $m_{A}$ and $m_{B}$ are obtained by solving the coupled Equations. (5) and (6). The results are depicted in Figure 4 for the system with $D_{A} / z|J|=1.0$, when the value of $D_{B} / z|J|$ is changed from $D_{B} / z|J|=-0.45$ to -1.05 . Notice that the selection of $D_{B} / z|J|$ corresponds to the crossover from the $O_{1}$ phase to the $O_{2}$ phase and from the $O_{2}$ to the $\mathrm{O}_{3}$ phase (see the ground-state phase diagram in Figure 1). Therefore, the ground state is always ordered and Figure 4 shows that the system undergoes only the second-order phase transition, because the sublattice magnetizations go to zero continuously as the temperature increases.

As shown in Figure 4, when $D_{B} / z|J|=-0.45$ (close to the boundary between the ordered-phase $O_{1}$ and the ordered phase $\mathrm{O}_{2}$ in the ground-state phase diagram), the temperature dependences of $m_{B}$ may exhibit a rather rapid decrease from its saturation value at $T=0 \mathrm{~K}$. The phenomena is further enhanced when the value of $D_{B} / z|J|$ approaches the boundary. At $D_{A} / z|J|=-0.5$ and for $T=0 \mathrm{~K}$, the saturation value of $m_{B}$ is $m_{B}=2.0$, which indicates that in the ground state the spin configuration of $S_{j}^{B}$ in the system consists of the mixed state; in this state half of the spins on sublattice $B$ are equal to

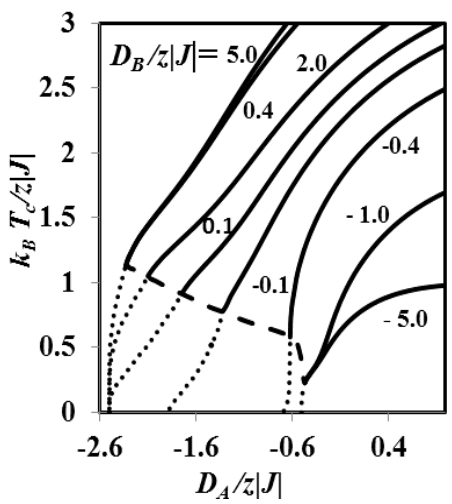

Figure 3. Phase diagram in the $\left(D_{A}, T\right)$ plane for the mixedspin Ising ferrimagnet with the coordination number $z$, when the value of $D_{B} / z|J|$ is changed. The solid and dotted lines, respectively, indicate second and first-order phase transitions, while the heavy dashed line represents the positions of tricritical points.

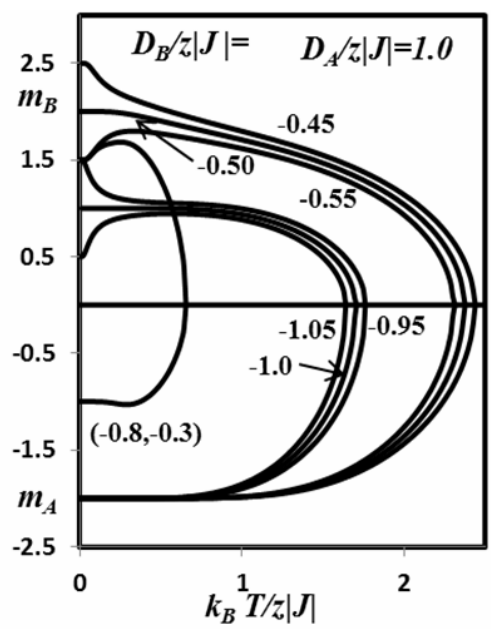

Figure 4. Thermal variations of sublattice magnetizations $m_{A}, m_{B}$ for the mixed-spin Ising ferrimagnet with the coordination number $z$, when the value of $D_{B} / z|J|$ is changed for fixed $D_{A} / z|J|=1.0$. For one curve $\left(D_{A} / z|J|, D_{B} / z|J|\right)=(-0.8$, $-0.3)$.

$+5 / 2$ (or $-5 / 2$ ) and the other half are equal to $+3 / 2$ (or $-3 / 2$ ). Note that this mixed state persists as long as $D_{B} / z|J|=-0.5$ and $D_{A} / z|J|>-0.5$.

In this case, the total magnetization for the ferrimagnetic system is $M=0$ at $T=0 \mathrm{~K}$, and hence, there is a compensation point at which the two sublattice magnetization cancel.

By further decreasing $D_{B} / z|J|$, the ground state becomes $O_{2}$, with $m_{B}=1.5$ at $T=0 \mathrm{~K}$. In this region, when $D_{B} / z|J|=-0.55$ (slightly below the boundary between the ordered phases $O_{1}$ and $O_{2}$ ) the thermal variation of $m_{B}$ exhibits an interesting feature which is the initial rise of $m_{B}$ with the increase of temperature before decreasing to zero at the critical point. On the other hand, for all values of $D_{B} / z|J|$, even though the sublattice 
magnetization $m_{A}$ may show normal behaviour it is coupled to $m_{B}$.

When $D_{B} / z|J|$ has the values $-0.95,-1.0$ and -1.05 (close to the end at the boundary between the orderedphases $\mathrm{O}_{2}$ and $\mathrm{O}_{3}$ in the ground-state phase diagram), it is clear from Figure 4 that the temperature dependences of $m_{B}$ and $m_{A}$ exhibit similar behaviours to the temperature dependences of $m_{B}$ and $m_{A}$ in the previous case.

At the point $\left(D_{A} / z|J|, D_{B} / z|J|\right)=(-0.8,-0.3)$, the system will be in the ordered phase $\mathrm{O}_{5}$ (see the groundstate phase diagram in Figure 1). In this case, the saturated values of $\left(m_{A}, m_{B}\right)$ are $(-1,3 / 2)$ at $T=0 \mathrm{~K}$. Notice that the sublattice magnetization $m_{B}$ has initial rise with temperature before decreasing to its zero value at the critical point, and the sublattice magnetization $m_{A}$ may show a normal behaviour with temperature.

\subsection{Compensation Temperature A}

Compensation temperature $T_{k}$ of the system can be evaluated by requiring the condition $M=0$; in Equation (13).

Figures 5(a) and (b) show the behaviour of $T_{k}$ (dotted lines) in the $\left(D_{B} / z|J|, k_{B} T / z|J|\right)$ plane for different values of $D_{A} / z|J|$. As seen from the figures, all $T_{k}$ curves emerge from $D_{B} / z|J|=-0.5$ at $T=0 \mathrm{~K}$ and exhibit some characteristic behaviours when the value of $D_{A} / z|J|$ is changed.

In Figure 5(a), all the curves increase monotonically with $D_{B} / z|J|$ and terminate at the corresponding phase boundaries (solid lines). This behaviour implies the occurrence of one compensation point only. As $D_{A} / z|J|$ is reduced, the range of $D_{B} / z|J|$ over which the compensation points occur gradually becomes small, but the compensation temperature still reaches the corresponding transition line. In the Figure 5(b), and in a restricted region of $D_{B} / z|J|$, close to $D_{B} / z|J|=-0.5$, a new type of compensation curves appear and the compensation temperature lines exhibit an interesting features in their behaviours, which implies the occurrence of two, three, or four compensation points. In this figure, for $D_{A} / z|J|$, close to $D_{A} / z|J|=-0.5$, a new type of compensation curves appear: the $T_{k}$ curves are extended to $D_{B} / z|J| \rightarrow-\infty$ below the corresponding transition lines. The curve labeled $D_{A} / z|J|=-0.498$ is an example of such behaviour of $T_{k}$. Finally, a total magnetization curve (which refers to the compensation temperatures presented in Figure 5(b)) when $D_{A} / z|J|=-0.498$ and $D_{B} / z|J|=-0.499222$ with four compensation points are shown in Figure 6. Furthermore, In Figure 7(a), when $D_{A} / z|J|=-0.4999$ and $D_{B} / z|J|=-0.5$ (very close to the point $\left(D_{A} / z|J|, D_{B} / z|J|\right)=(-0.5,-0.5)$ which is in the boundary between five phases in the ground state phase diagram), the magnetization curves

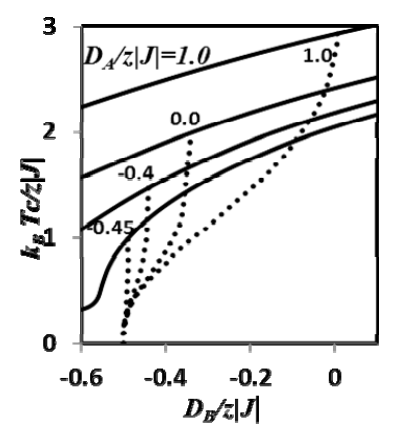

(a)

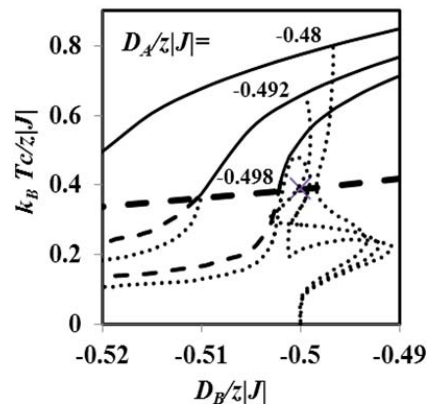

(b)
Figure 5. Dependence of the compensation temperature (dotted curves) on the single-ion anisotropy. $D_{B} / z|J|$ in a mixed-spin Ising ferrimagnet with coordination number $z$, when the value of $D_{B} / z|J|$ is changed. (a) The curves show the positions of one compensation points; (b) The curves show the positions of two, three and four compensation points. The solid and dashed curves represent the second and first-order transitions.

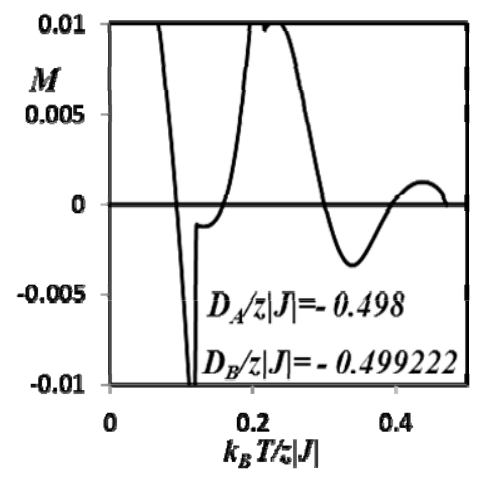

Figure 6. Thermal variations of the total magnetization $M$ for the mixed-spin Ising ferrimagnet with the coordination number $z$, when the value of $D_{A} / z|J|=-0.498$ and the value of $D_{B} / z|J|=-0.499222$.

exhibit some outstanding features. At this point, as the temperature is increased from zero, the sublattice magnetizations $m_{A}$ and $m_{B}$ exhibit four jumps (discontinuity) before the magnetizations vanish, indicating the existence of four first order transitions at the temperature values $k_{B} T / z|J|=0.0797,0.1583$ and 0.2526 respectively. In the same time, as shown in Figure 7(b), the total magnetization exhibits four first order transition points and four compensation temperatures.

\section{Conclusion}

In this paper, we have determined the global phase diagrams of the mixed spin-2 and spin-5/2 Ising ferrimagnetic system with different single-ion anisotropies acting on the spin- 2 and spin-5/2 by using mean-field approximation. In the phase diagrams, the critical temperature lines versus single-ion anisotropies are shown. The system presents tricritical behaviour, i.e., the second-order 


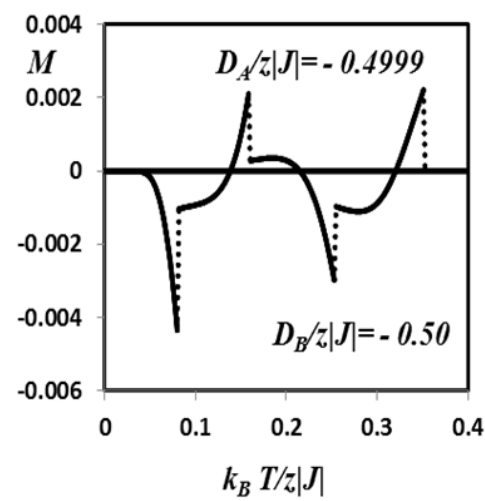

(a)

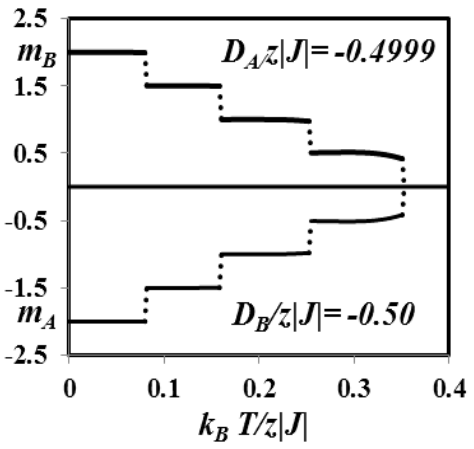

(b)

Figure 7. Thermal variations of (a) The total magnetization $M$; (b) The sublattice magnetizations $m_{A}, m_{B}$ for the mixedspin Ising ferrimagnet with the coordination number $z$, when the value of $D_{A} / z|J|=-0.4999$ and the value of $D_{B} / z|J|$ $=-\mathbf{0 . 5}$.

phase transition line is separated from the first-order transition line by a tricritical point. We also observed that this mixed-spin ferrimagnetic system may exhibit one, two, three or four compensation points. The theoretical prediction of the possibility of compensation points and the design and preparation of materials with such unusual behaviour will certainly open a new area of research on such materials.

\section{REFERENCES}

[1] O. Khan, "Molecular Magnetism," VCH publishers, New York, 1993.

[2] T. Kaneyoshi and Y. Nakamura, "A Theoretical Investigation for Low-Dimensional Molecular-Based Magnetic Materials," Journal of Physics: Condensed Matter, Vol. 10 , No. 13,1998 , p. 3003. doi:10.1088/0953-8984/10/13/017

[3] T. Kaneyoshi, Y. Nakamura and S. Shin, "A Diluted Mixed Spin-2 and Spin-5/2 Ferrimagnetic Ising System; a Study of a Molecular-Based Magnet," Journal of Physics: Condensed Matter, Vol. 10, No. 31, 1998, p. 7025. doi:10.1088/0953-8984/10/31/018

[4] L. Néel, "Propriétées Magnétiques des Ferrites; Férrimag- nétisme et Antiferromagnétisme," Annales de Physique (Paris), Vol. 3, 1948, pp. 137-198.

[5] M. Mansuripur, "Magnetization Reversal, Coercivity, and the Process of Thermomagnetic Recording in Thin Films of Amorphous Rare Earth Transition Metal Alloys," Journal of Applied Physics, Vol. 61, No. 4, 1987, pp. 15801587. doi:10.1063/1.338094

[6] F. Tanaka, S. Tanaka and N. Imamura, "Magneto-Optical Recording Characteristics of TbFeCo Media by Magnetic Field Modulation Method," Japan Journal of Applied Physics, Vol. 26, 1987, pp. 231-235. doi:10.1143/JJAP.26.231

[7] L. L. Goncaloves, "Uniaxial Anisotropy Effects in the Ising Model: An Exactly Soluble Model," Physica Scripta, Vol. 32, No. 3, 1985, p. 248. doi:10.1088/0031-8949/32/3/012

[8] L. L. Goncaloves, "Uniaxial Anisotropy Effects in the Ising Model: An Exactly Soluble Model," Physica Scripta, Vol. 33, No. 2, 1986, p. 192. doi:10.1088/0031-8949/33/2/018

[9] A. Dakhama and N. Benayad, "On the Existence of Compensation Temperature in $2 \mathrm{~d}$ Mixed-Spin Ising Ferrimagnets: An Exactly Solvable Model," Journal of Magnetism and Magnetic Materials, Vol. 213, No. 1-2, 2000, pp. 117-125. doi:10.1016/S0304-8853(99)00606-X

[10] N. R. da Silva and S. R. Salinas, "Mixed-Spin Ising Model on Beth Lattice," Physical Review, Vol. 44, No. 2, 1991, pp. 852-855. doi:10.1103/PhysRevB.44.852

[11] J. W. Tucker, "The Ferrimagnetic Mixed Spin-1/2 and Spin-1 Sing System," Journal of Magnetism and Magnetic Materials, Vol. 195, No. 3, 1999, pp. 733-740. doi:10.1016/S0304-8853(99)00302-9

[12] T. Kaneyoshi and J. C. Chen, "Mean-Field Analysis of a Ferrimagnetic Mixed Spin System," Journal of Magnetism and Magnetic Materials, Vol. 98, No. 1-2, 1991, pp. 201-204. doi:10.1016/0304-8853(91)90444-F

[13] T. Kaneyoshi, "Curie Temperatures and Tricritical Points in Mixed Ising Ferromagnetic Systems," The Physical Society of Japan, Vol. 56, 1987, pp. 2675-2680. doi:10.1143/JPSJ.56.2675

[14] T. Kaneyoshi, "Phase Transition of the Mixed Spin System with a Random Crystal Field," Physica A, Vol. 153, No. 3, 1988, pp. 556-566. doi:10.1016/0378-4371(88)90240-3

[15] T. Kaneyoshi, M. Jascur and P. Tomczak, "The Ferrimagnetic Mixed Spin-1/2 and Spin-3/2 Ising System," Journal of Physics: Condensed Matter, Vol. 4, No. 49, 1992, pp. L653-L658. doi:10.1088/0953-8984/4/49/002

[16] T. Kaneyoshi, "Tricritical Behavior of a Mixed Spin-1/2 and Spin-2 Ising System," Physica A, Vol. 205, No. 4, 1994, pp. 677-686. doi:10.1016/0378-4371(94)90229-1

[17] A. Bobak and M. Jurcisin, "Discussion of Critical Behaviour in a Mixed-Spin Ising Model," Physica A, Vol. 240, No. 3-4, 1997, pp. 647-656. doi:10.1016/S0378-4371(97)00044-7

[18] S. G. A. Quadros and S. R. Salinas, "RenormalizationGroup Calculations for a Mixed-Spin Ising Model," Physica A: Statistical Mechanics and Its Applications, Vol. 206, No. 3-4, 1994, pp. 479-496.

[19] G.-M. Zhang and C.-Z. Yang, "Monte Carlo Study of the 
Two-Dimensional Quadratic Ising Ferromagnet with Spins $\mathrm{S}=1 / 2$ and $\mathrm{S}=1$ and with Crystal-Field Interactions," Physical Review B, Vol. 48, No. 13, 1993, pp. 9452-9455. doi:10.1103/PhysRevB.48.9452

[20] G. M. Buendia and M. A. Novotny, "Numerical Study of a Mixed Ising Ferrimagnetic System," Journal of Physics: Condensed Matter, Vol. 9, No. 27, 1997, pp. 5951-5964. doi:10.1088/0953-8984/9/27/021

[21] G. M. Buendia and J. A. Liendo, "Monte Carlo Simulation of a Mixed Spin-1/2 and Spin-3/2 Ising Ferrimagnetic System," Journal of Physics: Condensed Matter, Vol. 9, No. 25, 1997, pp. 5439-5448. doi:10.1088/0953-8984/9/25/011

[22] A. Bobak, "The Effect of Anisotropies on the Magnetic Properties of a Mixed Spin-1 and Spin-3/2 Ising Ferrimagnetic System," Physica A, Vol. 258, No. 1-2, 1998, pp. 140-156. doi:10.1016/S0378-4371(98)00233-7

[23] O. F. Bobak and D. H. Abubrig, "An Effective-Field Study of the Mixed Spin-1 and Spin-3/2 Ising Ferrimagnetic System," Journal of Magnetism and Magnetic Materials, Vol. 246, No. 1-2, 2002, pp. 177-183. doi:10.1016/S0304-8853(02)00048-3

[24] O. F. Abubrig, D. Horvath, A. Bobak and M. Jascur, "Mean-Field Solution of the Mixed Spin-1 and Spin-3/2 Ising System with Different Single-Ion Anisotropies," Physica A, Vol. 296, No. 3-4, 2001, pp. 437-450. doi:10.1016/S0378-4371(01)00176-5

[25] J. W. Tucker, "Mixed Spin-1 and Spin-3/2 Blume-Capel Ising Ferromagnet," Journal of Magnetism and Mgnetic Materials, Vol. 237, No. 2, 2001, pp. 215-224. doi:10.1016/S0304-8853(01)00691-6

[26] Y. Nakamura and J. W. Tucker, "Monte Carlo Study of a Mixed Spin-1 and Spin-3/2 Ising Ferromagnet," IEEE Transactions on Magnetics, Vol. 38, No. 5, 2002, pp. 2406-2408. doi:10.1109/TMAG.2002.803598

[27] A. Bobak and J. Dely, "Phase Transitions and Multicritical Points in the Mixed Spin-3/2 and Spin-2 Ising System with a Single-Ion Anisotropy," Journal of Magnetism and Magnetic Materials, Vol. 310, No. 2, 2007, pp. 14191421. doi:10.1016/j.jmmm.2006.10.427

[28] E. Albayrak, "The Critical and Compensation Temperatures of the Mixed Spin-3/2 and Spin-2 Ising Model," Physica B: Condensed Matter, Vol. 391, No. 1, 2007, pp.
47-53. doi:10.1016/j.physb.2006.08.045

[29] B. Deviren, E. Kantar and M. Keskin, "Magnetic Properties of a Mixed Spin-3/2 and Spin-2 Ising Ferrimagnetic System within the Effective-Field Theory," Journal of the Korean Physical Society, Vol. 56, No. 6, 2010, pp. 17381747. doi:10.3938/jkps.56.1738

[30] Y. Nakamura, S. Shin and T. Kaneyoshi, "The Effects of Transverse Field on the Magnetic Properties in a Diluted Mixed Spin-2 and Spin-5/2 Ising System," Physica B, Vol. 284-288, 2000, pp. 1479-1480. doi:10.1016/S0921-4526(99)02668-X

[31] Y. Nakamura, "Monte Carlo Study of a Mixed Spin-2 and Spin-5/2 Ising System on a Honeycomb Lattice," Journal of Physics: Condensed Matter, Vol. 12, No. 17, 2000, pp. 4067-4074. doi:10.1088/0953-8984/12/17/312

[32] Y. Nakamura, "Existence of a Compensation Temperature of a Mixed Spin-2 and Spin-5/2 Ising Ferrimagnetic System on a Layered Honeycomb Lattice," Physical Review B, Vol. 62, No. 17, 2000, pp. 11742-11746. doi:10.1103/PhysRevB.62.11742

[33] J. Li, A. Du and G. Z. Wei, "Green Function Study of a Mixed-Spin-2 and Spin-5/2 Heisenberg Ferrimagnetic System on a Honeycomb Lattice," Physica Status Solidi (b), Vol. 238, No. 1, 2003, pp. 191-197.

[34] J. Li, A. Du and G. Z. Wei, "The Compensation Behavior of a Mixed-Spin-2 and Spin-5/2 Heisenberg Ferrimagnetic System on a Honeycomb Lattice," Physica B, Vol. 348 , No. 1-4, 2004, pp. 79-88. doi:10.1016/j.physb.2003.11.074

[35] G. Wei, Q. Zhang, Z. Xin and Y. Liang, "Internal Energy and Initial Susceptibility of Mixed Spin-2 and Spin-5/2 Ferrimagnetic Ising System with Interlayer Coupling," Journal of Magnetism and Magnetic Materials, Vol. 277, No 1-2, 2004, pp. 1-15. doi:10.1016/j.jmmm.2003.06.001

[36] E. Albayrak, "Mixed-Spin-2 and Spin-5/2 Blume-EmeryGriffiths Model," Physica A: Statistical Mechanics and Its Applications, Vol. 375, No. 1, 2007, pp. 174-184.

[37] M. Keskin and M. Ertas, "Existence of a Dynamic Compensation Temperature of a Mixed Spin-2 and Spin-5/2 Ising Ferrimagnetic System in an Oscillating Field," Physical Review E, Vol. 80, No. 6, 2009, Article ID: 061140. doi:10.1103/PhysRevE.80.061140 